\title{
Investigation of microplastics removal methods from aquatic environments
}

\author{
Sevgi Gunes-Durak ${ }^{1^{*}}$ \\ ${ }^{1}$ Faculty of Engineering and Architecture, Department of Environmental Engineering, Nevsehir Haci Bektas Veli University, Turkey
}

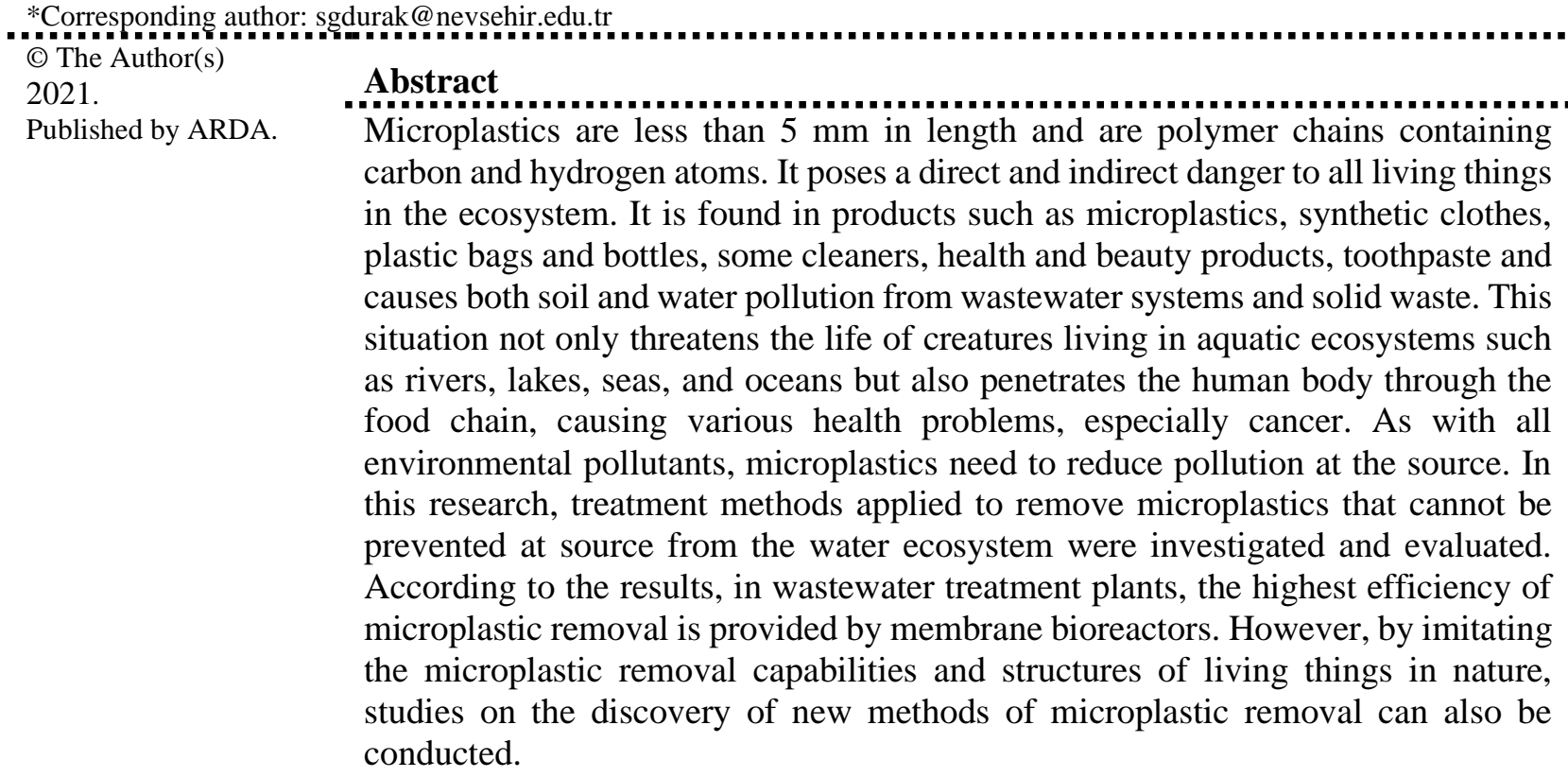

Keywords: Activated sludge, Sand filter, Membrane bioreactor, Microplastics

\section{Introduction}

Plastics are synthetic or semi-synthetic organic components that we encounter at every point of our daily life from textiles to cosmetics, from furniture to packaging [1]. It poses a problem both physically and toxic for the environment and living things as it contains phthalate, bisphenol A and polybrominated diphenyl ether. However, microplastics (MPs) formed by breaking down smaller sized plastics by different methods can create bigger problems for living things such as accumulation, immune response, respiratory problems and chemical leaching [2]. Besides, they cause slow growth and reproduction of algae in aquatic environments, structural deformation of water fleas, and obstruction of the respiratory tract of gill marine creatures.

MPs can be of different sizes, different types, and different densities. The transformation of plastics into MPs takes place with anthropogenic effects, natural effects such as water, air, wind, sun (UV). As they can be in a primary form such as direct use (personal care products $(1 \mathrm{~mm}-4 \mathrm{~mm}$ microbeads), scrubs, toothpaste, industrial raw materials) or indirect use (pellets); it can also be in secondary forms such as car tires, boat paint, etc. formed by fragmentation of larger plastic items, fibers from washing machines.

MPs are pollutants that degrade slowly in water and soil. It has a long residence time, high stability, high disintegration potential in their environment, and can adsorb other pollutants [3]. Since their density is less than water, they move on the water surface. It has been stated that in deep-sea sediments there is 1 MP in every 25 $\mathrm{mL}$, microplastics are found even at a depth of $1176-2843 \mathrm{~m} \mathrm{[4].} \mathrm{The} \mathrm{fact} \mathrm{that} \mathrm{MPs} \mathrm{consist} \mathrm{of} \mathrm{smaller} \mathrm{particles}$ creates a disadvantage in removal. Also, it is not possible to see colorless transparent MPs in the aquatic environment unless they are looked at carefully. However, MPs can be transported from one end of the world

This work is licensed under a Creative Commons Attribution License (https://creativecommons.org/licenses/by/4.0/) that allows others to share and adapt the material for any purpose (even commercially), in any medium with an acknowledgement of the work's authorship and initial publication in this journal. 
to the other by winds and ocean currents. As a result of not disposing of wastes by appropriate methods such as landfills, MPs spread to soil and water resources. They pose a higher risk of adding to the food chain than plastic materials. It is an important problem that it is taken into the organism voluntarily or unintentionally by aquatic creatures by mixing with the soil structure and the water environment. Living things that feed on very small pieces of food, feel their stomachs full and die as a result of malnutrition [5]. In addition to breaking down plastics, resins in the form of pellets are produced as industrial raw materials. Pellets are melted in industrial facilities and transformed into products such as plastic bottles, caps, bags and packaging. Production debris that may occur during the transport and processing of these pellets is also a source of MP pollution. These microplastics are also mixed into the food chain by passing into the water environment through the sewage system. MPs are found in nature as primary MPs as household personal care products and industrial raw materials and secondary MPs by degrading by physical, chemical and biological processes in the environment [6].

In this study, the number of microplastics found in wastewater treatment plants and the treatment methods used to remove microplastics in the literature were investigated and some methods used in microplastic characterization were mentioned.

\section{Microplastics}

\subsection{Classification of microplastics}

MPs are classified according to their physical and chemical characters. Physical characterization gives size distribution and shows shape and color properties. Chemical characterization allows us to discover the composition of MPs [7].

According to their dimensions, they are divided into three classes as nano plastics $(<1 \mu \mathrm{m})$, microplastics $(<5$ $\mathrm{mm}$ ) and mesoplastics (>5 mm) [8]. According to their shapes, they are divided into six classes as a particle (hard and sharp plastic particles), film (thin and light surface particles), foam (thin and light foam plastic particles), fiber (thin and light plastic particles), yarn (fine and durable fiber particles), pellet (hard and round plastic particles) [9], [10]. The size of MPs is important in terms of their removal from wastewater treatment plants. While plastic wastes are removed from the wastewater with coarse filters, MPs with a size smaller than $5 \mathrm{~mm}$ can be discharged into aquatic environments by passing through the treatment unit. However, MPs, which are formed as a result of the breakdown of colorless plastics, are difficult to detect compared to colored MPs, since they are invisible in an aquatic environment, requiring first filtering using a very fine sieve and then classifying shape, color and size with a microscope [10]. Advanced treatment methods should be determined according to the results obtained.

\section{Removal methods of MPs}

\subsection{Natural removal of MPs in nature}

MPs can be kept naturally by the creatures in the water and removed from the water environment. The method of removal carried out in this way is called sorption. The biological treatment of MPs is based on sludge consumption by microorganisms. It exhibits sludge hydrolysis, acetic acid accumulation, hydrogen production and methane production.

Studies have shown that a microalga called Fucus Vesiculosus holds microplastics of about $20 \mu \mathrm{m}$ in size. This adhesion was achieved with alginate compounds released from cell walls [11]. Alginate, an anionic polysaccharide substance, enables polystyrene type MPs to stick to the seaweed surface. Pseudokirchneriella Subcapitata, another green alga, keeps positively charged microplastics on its surface [12]. This adhesion depends on the surface load of the particles attached to the algae. If MPs are positively charged, they provide more effective adhesion depending on the presence of anionic polysaccharides in algae chemical structure. Ingestion of MPs by organisms is still not considered a disposal strategy, but Red Sea giant oysters allow MPs to be absorbed in shells as well as degradation in the digestive system [3].

\subsection{MPs in wastewater treatment plants}

Since MPs are small in size and their types vary, it is not possible to purify only from a coarse filter in wastewater treatment plants (WWTP). Therefore, advanced technologies are needed in treatment. However, MPs constitute a large part of the wastes in WWTP [13], [14]. 30 types of MPs have been identified in WWTP so far. The most detected type is polyethylene sulfone (PES) with $89 \%$ (Table 1) [10]. 
Table 1. According to the results of 42 studies, the most common types of MPs in the aquatic environment [10]

\begin{tabular}{lc}
\hline Polymer type & \% of studies (n) \\
\hline Polyethylene (PE) & $79(33)$ \\
Polypropylene (PP) & $64(27)$ \\
Polystyrene (PS) & $40(17)$ \\
Polyamide (nylon) (PA) & $17(7)$ \\
Polyester (PES) & $10(4)$ \\
Acrylic (AC) & $10(4)$ \\
Polyoxymethylene (POM) & $10(4)$ \\
Polyvinyl alcohol (PVA) & $7(3)$ \\
Polyvinyl chloride (PVC) & $5(2)$ \\
Polymethyl acrylate (PMA) & $5(2)$ \\
Polyethylene terephthalate (PET) & $2(1)$ \\
Alkyd (AKD) & $2(1)$ \\
Polyurethane (PU) & $2(1)$ \\
\hline
\end{tabular}

Physical treatment methods such as advanced filtration, biological treatment and chemical treatment methods are applied for the removal of microplastics in wastewater. In the physical treatment, aeration tank [3], [15], sedimentation tank [3], [15], [16], flotation [3] and filtration [3] methods; in the chemical treatment, coagulation and agglomeration using $\mathrm{Fe}^{3+}$ and $\mathrm{Al}^{3+}$ ions [17], [18], $\mathrm{TiO}_{2}, \mathrm{ZnO}, \mathrm{NaI}$ ve $\mathrm{ZnCl}$ ions [7]; in the biological treatment, conventional active sludge, $\mathrm{A}^{2} \mathrm{O}$ (anoxic, aerobic, anaerobic) methods [16], [19] and in advanced treatment, denitrification, ultrafiltration, ozone and UV methods [16], [18], [19] were used frequently. In a wastewater treatment plant study, consisting of influent, grit and grease removal unit, primary settling tank and aeration basin, clarification and outfall units, respectively, the daily and annual microplastic amounts from each unit and the percentage MP removal rates from each unit are given in Table 2.

Table 2. MP removal amounts and rates in a wastewater treatment plant [20]

\begin{tabular}{ccccc}
\hline Units & MP/L & Million MP/Day & Million MP/Year & \% Removal \\
\hline Influent & 15.70 & 4,097 & $1,495,397$ & 0.00 \\
Grit and grease removal & 8.70 & 2,270 & 828,559 & 44.59 \\
Primary settling tank & 3.40 & 887 & 323,844 & 78.34 \\
Aeration basin+ clarification + outfall & 0.25 & 65 & 23,812 & 98.41 \\
\hline
\end{tabular}

In another study by Talvitie et al. conducted the rate of MPs removed in each unit is given in Table 3 [19]. According to Table 3, MP removal efficiency in wastewater treated separately from disc filters, rapid sand filter, dissolved air flotation and membrane bioreactor has reached up to $99.9 \%$ in treatment with membrane bioreactor (MBR). In another study, a dynamic membrane was used for the removal of MPs, but it was stated that new methods should be developed in terms of energy [21].

Table 3. MP removal amounts and rates in a WWTP [19]

\begin{tabular}{llccc}
\hline Treatment & Effluent type & Before (MP/L) & After (MP/L) & Removal (\%) \\
\hline Disk filter $10^{\mathrm{a}}$ & Secondary & 0.5 & 0.3 & 40.0 \\
Disk filter $20^{\mathrm{a}}$ & Secondary & 2.0 & 0.03 & 98.5 \\
Rapid sand filter & Secondary & 0.7 & 0.02 & 97.1 \\
Dissolved air flotation & Secondary & 2.0 & 0.1 & 95.0 \\
Membrane bioreactor & Primary & 6.9 & 0.005 & 99.9 \\
\hline
\end{tabular}

Data are given in the number of microplastics per liter of effluent

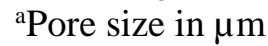




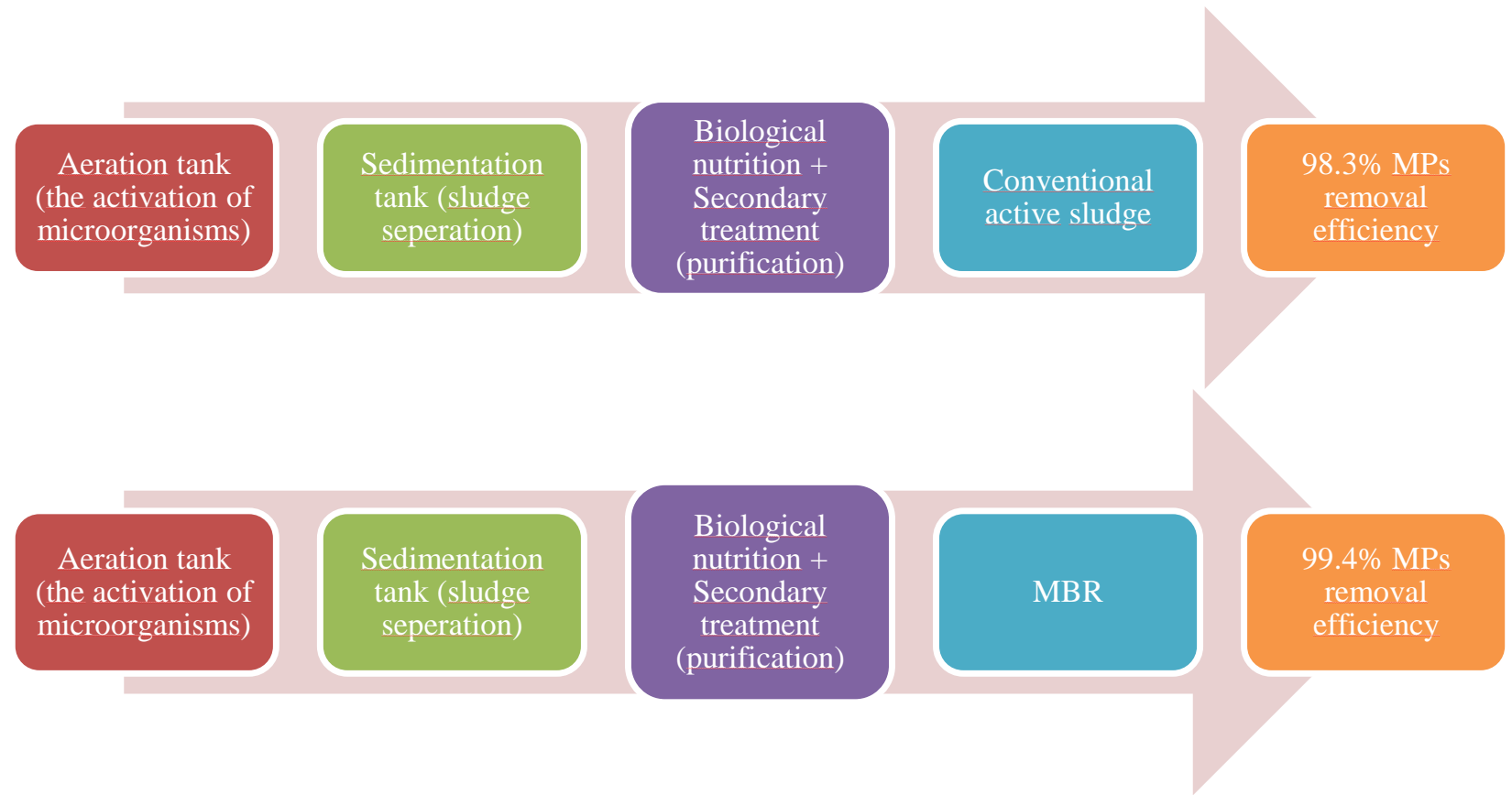

Figure 1. Comparison of removal of MPs in different wastewater treatment units

In a study by Gurung et al. conducted by wastewater regarding the removal of MPs from wastewater, removal efficiency of $98.3 \%$ was determined when the last treatment unit was conventional active sludge, while this removal efficiency increased to $99.4 \%$ when the last unit was MBR (Figure 1) [15].

In China, $90 \%$ of MPs removal was achieved when flotation and sedimentation + filtration were applied. The removal efficiency at the exit of the facility has been determined as $97.15 \%$. The most removed MPs were determined as fiber rayon and polyethylene terephthalate [3].

In another MP treatment study in China, with the aerated grit chamber, primary and secondary precipitation such as anaerobic, anoxic and aerobic treatment following $\mathrm{A}^{2} \mathrm{O}$ treatment were used. Finally, MP removal and the cycle is completed with advanced treatment technologies including denitrification, ultrafiltration, ozonation and UV. The most abundant MP in wastewater was determined as polyethylene terephthalate with $42.26 \%$. While 58.84\% MP was removed in the first treatment step in the aerated sand chamber, this rate reached $71.67 \%$ in further treatment [16]. This rate has been determined as $95.16 \%$ in WWTP and $99.9 \%$ after the MBR unit [22].

In a study conducted by Ma et al, chemical treatment of MPs was carried out using $\mathrm{Al}^{3+}$ and $\mathrm{Fe}^{3+}$ salts. Polyacrylamide (PAM) was also added in the treatment unit and it was observed that the treatment efficiency was enhanced. However, it was found that $\mathrm{Al}^{3+}$ ions are more effective than $\mathrm{Fe}^{3+}$ ions [3], [18]. It was seen that removal efficiency was affected by factors such as coagulant type, $\mathrm{pH}$, chemical composition and concentration. In another study, MPs are separated from synthetic wastewater by membranes. The membrane pore diameter was $90 \mu \mathrm{m}$. The turbidity of wastewater has been reduced from 195 NTU to less than 1 NTU in 20 minutes [3]. However, MBRs provide higher removal capacity. 99.9\% removal efficiency has been achieved with the combination of the porous membrane.

\section{MPs analysis methods in wastewater}

FTIR is the most widely used method for MPs characterization in WWTPs. It gives an idea of MP composition through to its wavelength. FTIR and electron microscopy analyzes are widely used to illuminate any structural changes during the degradation process. The observation of hydroperoxide and hydroxyl groups, carbonyl groups and double bond characteristic bands in FTIR models of treated MPs suggests the oxidative mechanism for biological removal [1].

Raman Spectroscopy, one of the most frequently used methods in MP characterization, provides information in the form of a vibration spectrum with molecular vibrations in the structure of MP [23]. Raman Spectroscopy can be applied to a wide variety of particle sizes up to $1 \mathrm{~mm}$ with existing instrumentation and provides a robust method for describing chemical composition. Also, Raman Spectroscopy-based microscopy techniques offer the possibility to place MPs even at the subcellular level in living organisms [1]. However, the requirement to 
purify the samples poses a disadvantage for Raman Spectroscopy.

Another method is Scanning Electron Microscopy with Energy Dispersive Spectroscopy (SEM-EDS) analysis. It can be used to provide information about the surface morphology of MPs and determine the basic composition of polymers based on the radiation diffraction and reflection emitted from the MP surface. SEM-EDS has become an indispensable tool for the identification and characterization of MP particles. This powerful technique has led researchers to conclude that the early stages of plastic debris degradation progress quite rapidly to the production of microplastic particles, with the resulting pollutants, clearly threatening biota life [1].

\section{Conclusion}

When sorption and filtration are combined with MBR, a high rate of purification is provided. Conventional activated sludge provides lower purification than MBR. However, the energy requirement for MBR leads to the investigation of alternative methods. Electrocoagulation and agglomeration are also reliable methods for easy separation but must have a filtration treatment. Alternative treatment methods for MPs removal can be determined by examining the mechanisms of marine organisms to keep MPs in their bodies and remove them from water.

[1] T. A. P. Rocha-Santos and A. C. Duarte, Characterization and Analysis of Microplastics, 1 st ed. Elsevier, 2017.

[2] A. A.; Horton, A.; Walton, and D. J.; Spurgeon, "Microplastics in freshwater and terrestrial environments: evaluating the current understanding to identify the knowledge gaps and future research priorities.," Sci. Total Environ., no. 586, pp. 127-141, 2017, doi: 10.1016/j.scitotenv.2017.01.190.

[3] M. Padervand, E. Lichtfouse, D. Robert, and C. Wang, "Removal of microplastics from the environment. A review," Environ. Chem. Lett., vol. 18, no. 3, pp. 807-828, May 2020, doi: 10.1007/s10311-02000983-1.

[4] L. Van Cauwenberghe, A. Vanreusel, J. Mees, and C. R. Janssen, "Microplastic pollution in deep-sea sediments," Environ. Pollut., vol. 182, pp. 495-499, 2013, doi: 10.1016/j.envpol.2013.08.013.

[5] E. Besseling, B. Wang, M. Lürling, and A. A. Koelmans, "Nanoplastic affects growth of S. obliquus and reproduction of D. magna," Environ. Sci. Technol., vol. 48, no. 20, pp. 12336-12343, Oct. 2014, doi: 10.1021/es503001d.

[6] F. Galgani, G. Hanke, S. Werner, and L. De Vrees, "Marine litter within the European Marine Strategy Framework Directive," ICES Journal of Marine Science, vol. 70, no. 6. pp. 1055-1064, Sep. 2013, doi: 10.1093/icesjms/fst122.

[7] J. Sun, X. Dai, Q. Wang, M. C. M. van Loosdrecht, and B. J. Ni, "Microplastics in wastewater treatment plants: Detection, occurrence and removal," Water Res., vol. 152, pp. 21-37, Apr. 2019, doi: 10.1016/j.watres.2018.12.050.

[8] M. Yurtsever, "Mikroplastikler'e Genel Bir Bakış (Microplastics: An Overview)," Dokuz Eylül Üniversitesi Mühendislik Fakültesi Fen ve Mühendislik Derg., vol. 17, no. 50, pp. 68-83, May 2015.

[9] K. Magnusson et al., "Swedish sources and pathways for microplastics to the marine environment," IVL Swedish Environ. Res. Inst., vol. C 183, 2016.

[10] V. Hidalgo-Ruz, L. Gutow, R. C. Thompson, and M. Thiel, "Microplastics in the marine environment: A review of the methods used for identification and quantification," Environ. Sci. Technol., vol. 46, no. 6, pp. 3060-3075, Mar. 2012, doi: 10.1021/es2031505.

[11] K. B. Sundbæk, I. D. W. Koch, C. G. Villaro, N. S. Rasmussen, S. L. Holdt, and N. B. Hartmann, "Sorption of fluorescent polystyrene microplastic particles to edible seaweed Fucus vesiculosus," $J$. Appl. Phycol., vol. 30, no. 5, pp. 2923-2927, Oct. 2018, doi: 10.1007/s10811-018-1472-8.

[12] T. M. Nolte et al., "The toxicity of plastic nanoparticles to green algae as influenced by surface modification, medium hardness and cellular adsorption," Aquat. Toxicol., vol. 183, pp. 11-20, Feb. 2017, doi: 10.1016/j.aquatox.2016.12.005.

[13] M. A. Browne et al., "Accumulation of microplastic on shorelines woldwide: Sources and sinks," Environ. Sci. Technol., vol. 45, no. 21, pp. 9175-9179, Nov. 2011, doi: 10.1021/es201811s.

[14] Z. Long et al., "Microplastic abundance, characteristics, and removal in wastewater treatment plants in a coastal city of China," Water Res., vol. 155, pp. 255-265, May 2019, doi: 10.1016/j.watres.2019.02.028. 
[15] K. Gurung, M. C. Ncibi, and J.-M. Fontmorin, "Incorporating Submerged MBR in Conventional Activated Sludge Process for Municipal Wastewater Treatment: A Feasibility and Performance Assessment," 2016, doi: 10.4172/2155-9589.1000158.

[16] L. Yang, K. Li, S. Cui, Y. Kang, L. An, and K. Lei, "Removal of microplastics in municipal sewage from China's largest water reclamation plant," Water Res., vol. 155, pp. 175-181, May 2019, doi: 10.1016/j.watres.2019.02.046.

[17] M. C. Ariza-Tarazona, J. F. Villarreal-Chiu, V. Barbieri, C. Siligardi, and E. I. Cedillo-González, "New strategy for microplastic degradation: Green photocatalysis using a protein-based porous N-TiO 2 semiconductor," Ceram. Int., vol. 45, no. 7, pp. 9618-9624, May 2019, doi: 10.1016/j.ceramint.2018.10.208.

[18] B. Ma, W. Xue, Y. Ding, C. Hu, H. Liu, and J. Qu, "Removal characteristics of microplastics by Febased coagulants during drinking water treatment," J. Environ. Sci. (China), vol. 78, pp. 267-275, Apr. 2019, doi: 10.1016/j.jes.2018.10.006.

[19] J. Talvitie, A. Mikola, A. Koistinen, and O. Setälä, "Solutions to microplastic pollution - Removal of microplastics from wastewater effluent with advanced wastewater treatment technologies," Water Res., vol. 123, pp. 401-407, Oct. 2017, doi: 10.1016/j.watres.2017.07.005.

[20] F. Murphy, C. Ewins, F. Carbonnier, and B. Quinn, "Wastewater Treatment Works (WwTW) as a Source of Microplastics in the Aquatic Environment," Environ. Sci. Technol., vol. 50, no. 11, pp. 5800-5808, Jun. 2016, doi: 10.1021/acs.est.5b05416.

[21] L. Li, G. Xu, H. Yu, and J. Xing, "Dynamic membrane for micro-particle removal in wastewater treatment: Performance and influencing factors," Sci. Total Environ., vol. 627, pp. 332-340, Jun. 2018, doi: 10.1016/j.scitotenv.2018.01.239.

[22] J. Talvitie, A. Mikola, O. Setälä, M. Heinonen, and A. Koistinen, "How well is microlitter purified from wastewater? - A detailed study on the stepwise removal of microlitter in a tertiary level wastewater treatment plant," Water Res., vol. 109, pp. 164-172, Feb. 2017, doi: 10.1016/j.watres.2016.11.046.

[23] D. Schymanski, C. Goldbeck, H. U. Humpf, and P. Fürst, "Analysis of microplastics in water by microRaman spectroscopy: Release of plastic particles from different packaging into mineral water," Water Res., vol. 129, pp. 154-162, Feb. 2018, doi: 10.1016/j.watres.2017.11.011. 